\title{
Development of a UHF Transponder for Geological Monitoring of Boreholes Drilled through Ice Sheets using phase-sensitive FMCW Radar
}

\author{
Amin Amiri, Paul V. Brennan, Lai Bun Lok \\ University College London, Department of Electronic and Electrical Engineering, London, UK
}

\begin{abstract}
This paper presents an active UHF transponder designed for geological monitoring of boreholes drilled through ice sheets. It forms part of a phase-sensitive frequency modulated continuous wave (FMCW) radar system to measure the horizontal position of a borehole with depth. To distinguish the transponder response from stationary clutter, the transponder modulates the received signal before re-transmission to the surface radars. The transponder operates from 292 to $492 \mathrm{MHz}$ with a gain around 18 dB. The transponder employs two novel antennas optimized for deployment within a $15 \mathrm{~cm}$ diameter borehole. The simulation and indoor laboratory measurement results of the transponder design are presented.
\end{abstract}

Index Terms-frequency modulated continuous wave (FMCW) radar, transponder, folded crossed plate monopole, ultrawideband (UWB) antenna

\section{INTRODUCTION}

Modern hot-water drilling provides a means of making boreholes rapidly and cleanly, to enable studies of the oceanographic conditions beneath ice shelves [1] and in subglacial lakes. Hot-water drilled boreholes also allow the ice column to be studied by freezing-in of instruments such as tilt meters and temperature sensors [2].

Within the glaciology research community, the horizontal position of hot-water drilled boreholes need to be known to submetre accuracy for two main scientific reasons. A pressure sensor at the bottom of a borehole is used to record the effect of a pulse of water released when a second borehole penetrates through the ice. In order to calculate substrate permeability, an outstanding requirement is to measure the distance between two boreholes at the ice-bed interface. The second need is to measure the amount of ice stream motion taken up as vertical shear in the ice column. One hypothesis is that this is likely to be around $1 \%$ of the total glacier motion, which would amount to a differential speed (top to bottom) of a few metres per year. To test this, one can monitor the changing tilt through the ice column using a daisy-chain of tilt meters deployed within the borehole. An independent measure can be obtained using the system proposed here by monitoring the changing location of the bottom of the borehole relative to the upper surface of the ice stream.

\section{PHASE-SENSITIVE RADAR WITH ACTIVE TRANSPONDER}

This paper proposes the development of a radar-transponder system that aims to give a precise, independent measure of how the horizontal location of a borehole changes with depth. In addition, the system allows the change in position (relative to the ice surface) of a given point in the borehole, such as at the ice base, to be monitored.

Ground-based phase sensitive radar with glaciological applications has been around since 1970s. Recently, phase-sensitive frequency modulated continuous wave (FMCW) radar has been applied for ice-shelf monitoring to measure vertical strain rates and time-varying basal melt rates in ice sheets and shelves to depths over $3 \mathrm{~km}$ with millimeter precision [3]. This measurement capability of phase-sensitive FMCW radar sets the foundation for the design of the proposed system in this work.

\section{A. Proposed Technique}

The setup for locating an active transponder is illustrated in Fig. 1, where three downward-looking phase-sensitive FMCW radars $(\mathrm{n} 1 \ldots \mathrm{n} 3)$ are deployed on the ice surface at a radius, $r$, from the centre of the borehole. For clarity, only one active transponder is shown deployed down a borehole and the position of that transponder is to be obtained by triangulation of three individual range measurements $R_{1} . . R_{3}[4]$.

The transponder is required to establish a link to the surface radar nodes through thick ice sheets and water cavities. The dimensions of the transponder antennas are limited by the borehole geometry. Many environmental and system requirements were considered during the antenna design process. It is assumed that the transponder is to operate, very soon after hotwater drilling, in an environment that is a few degrees above freezing.

A block diagram of the phase-sensitive FMCW radar and transponder is given in Fig. 2. The radar uses a direct digital synthesiser (DDS) chirp generator driven by a high stability 10 $\mathrm{MHz}$ clock. An in-phase quadrature $(I Q)$ deramping technique is used to cancel the unwanted effect of phase fluctuations of the free-running oscillator in the frequency-shifted active transponder. Conveniently, this approach also improves the receiver noise figure, and therefore the overall system sensitivity, by an additional $3 \mathrm{~dB}$. 


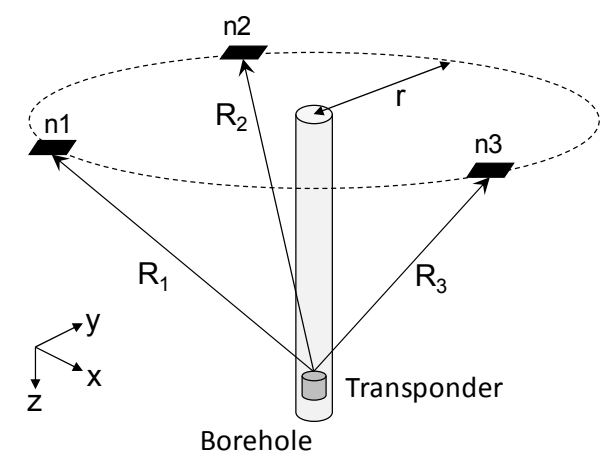

Fig. 1. Illustration of the borehole position measurement concept.

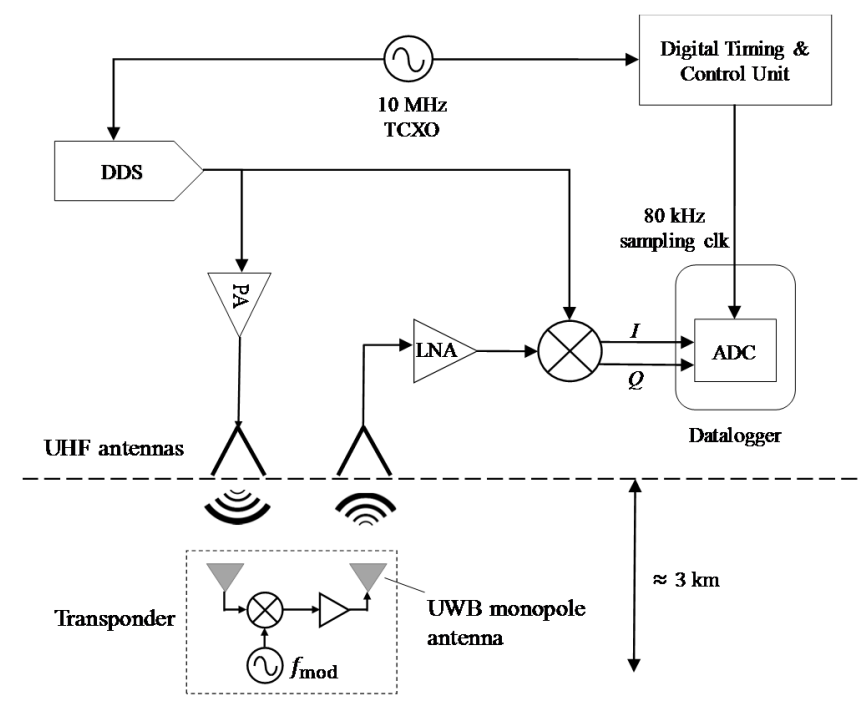

Fig. 2. Phase-sensitive FMCW radar and frequency shifted active transponder.

The active transponder, shown in Fig. 2, consists of a frequency-shifted RF chain and two UHF ultra-wideband (UWB) monopole antennas for reception and re-transmission of the radar chirp signal. This technique shifts the deramped echo from an active transponder to upper and lower sidebands around a higher frequency $f_{\text {mod }}$ thereby minimising the effect of close-in clutter noise and undesirable echoes from nearby reflectors surrounding the transponder [5]. The actual value of $f_{\text {mod }}$ is noncritical and different transponders can be distinguished using different $f_{\text {mod }}$ values. A typical value around $8 \mathrm{kHz}$ would still allow a 16-bit analogue-to-digital converter (ADC) with modest sampling rate to be used for the datalogger. A dual-channel $\mathrm{ADC}$ is required to capture the $I Q$ deramped signals for postprocessing to cancel the effect of random phase fluctuations within the transponder, which would otherwise degrade the range measurement precision.

\section{TRANSPONDER DESIGN}

\section{A. RF electronics unit}

The transponder RF electronics unit is designed and implemented using commercial off-the-shelf components. Fig. 3(a) depicts its schematic. The circuit is fabricated on a Rogers RO4350B substrate (relative permittivity $\varepsilon_{r}=3.48$ ) as shown in Fig. 3(b). The unit operates between $292 \mathrm{MHz}$ to $492 \mathrm{MHz}$. The received signal, after being filtered and amplified, is modulated with a clock frequency $f_{\text {mod. }}$. A square wave signal of constant frequency $f_{\text {mod }}=8 \mathrm{kHz}$ is generated by a low power, programmable waveform generator (AD9833, Analog Devices Inc.). The overall gain of the transponder is chosen so it does not exceed the coupling or isolation between the two antennas. The antenna coupling or isolation level is controlled by varying the distance between the two antennas. The total current consumption of the transponder is $120 \mathrm{~mA}$ from a $5 \mathrm{~V}$ battery power supply. Fig. 4 shows the scattering parameters of the transponder measured with a vector network analyser after short-open-load-thru calibration. The forward transmission gain is around $18 \mathrm{~dB}$ across the operating frequency range. (a)

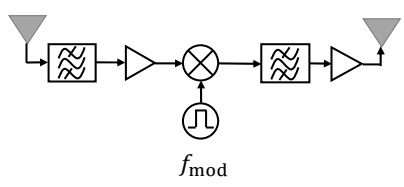

(b)

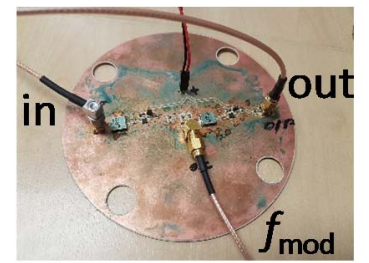

Fig. 3. (a) Schematic of the frequency shifted active transponder, and (b) photograph of the fabricated RF electronics unit.

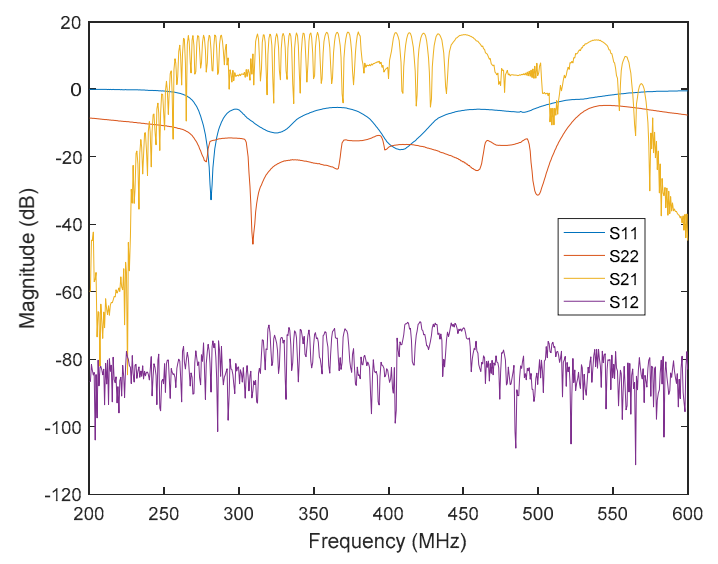

Fig. 4. Measured scattering parameters of the transponder RF electronics unit with port 1 as the input and port 2 as the output. 


\section{B. UWB monopole antenna}

The transponder must fit within a maximum borehole diameter of $15 \mathrm{~cm}$, which is only a very small portion of a wavelength at the intended operating frequencies. In addition, the complete transponder must be housed in a waterproof structure. Dipole antennas are commonly adopted for borehole radars because they are thin and long in shape. However, the radiation pattern of dipole antennas is usually omnidirectional in the azimuth plane with maximum directivity at boresight direction. The maximum boresight directivity limits the amount of signal that can reach the surface. The novel antenna design proposed here provides an omnidirectional heart-shape radiation pattern with the maximum signal directivity at an angle sharper than boresight.

Fig. 5 shows the modelled and fabricated structure of the proposed transponder. The implementation of the transponder consists of a cylindrical housing with rounded top and bottom caps. The housing accommodates the transponder electronics and antennas. It is $120 \mathrm{~cm}$ long and $15 \mathrm{~cm}$ in diameter. Careful design considerations were taken on transponder unit size, weight, water resistance and mechanical stability to ensure compatibility with typical borehole parameters. The transponder antennas are based on UWB folded crossed plate monopole design. The antennas can have impedance matching better than $10 \mathrm{~dB}$ from $190 \mathrm{MHz}$ to $1 \mathrm{GHz}$. However, the structural parameters of the antenna and transponder are optimised to achieve the best performance over the frequency range of $292 \mathrm{MHz}$ to $492 \mathrm{MHz}$. The gain of the antenna varies from $2 \mathrm{~dB}$ at lower frequency band to $4 \mathrm{~dB}$ at higher frequency band. The overall efficiency of the antenna is $95 \%$ at center frequency. The main direction of the signal reception and transmission of the transponder is located between 30 to $45^{\circ}$ from vertical alignment of the transponder.

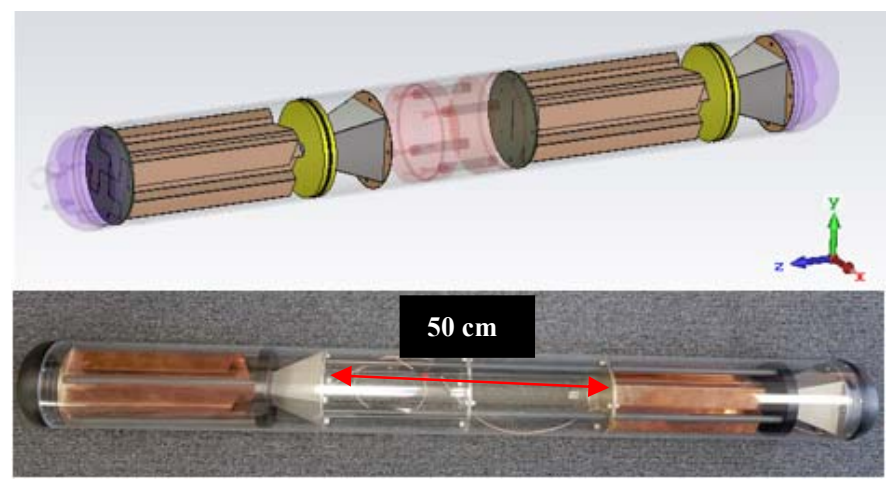

Fig. 5. The modelled and fabricated transponder structure in its housing.

The simulated and measured frequency response of the proposed antennas are shown in Fig. 6. The measured results were obtained in a non-ideal indoor environment. Nevertheless, the two antennas show an isolation predicted better than $20 \mathrm{~dB}$ with a distance of $50 \mathrm{~cm}$ between them. A good isolation between the antennas ensures negligible crosstalk and self-oscillation.

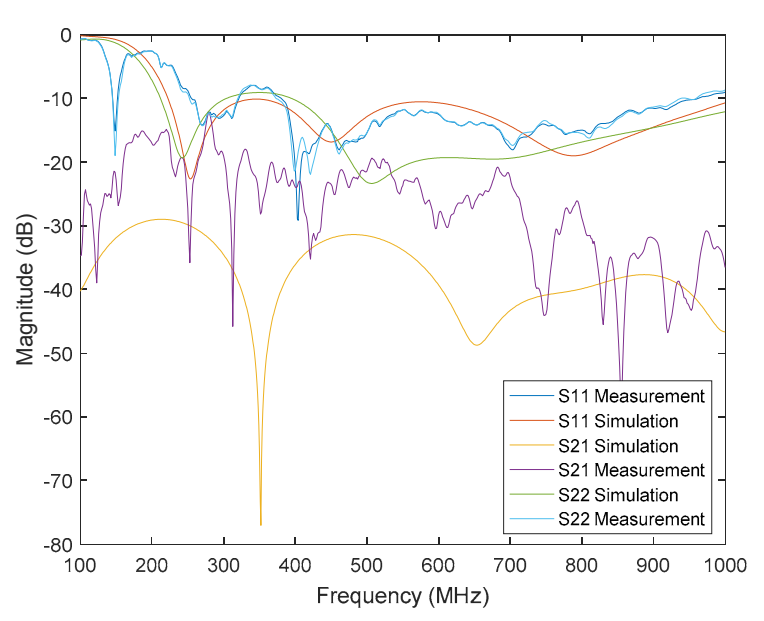

Fig. 6. Simulated and measured frequency response of the antennas separated by $50 \mathrm{~cm}$ inside the housing. Transmit antenna on port 1 , Receive antenna on port 2 .

\section{EXPERIMENTAL RESULTS}

Measurements in an indoor environment with surrounding clutter objects were performed using the prototype transponder and a phase-sensitive FMCW radar connected to two cavitybacked bow-tie antennas. The nominal transmit power is 20 $\mathrm{dBm}$ and the chirp sweeps from $200 \mathrm{MHz}$ to $400 \mathrm{MHz}$ over a duration of $1 \mathrm{~s}$. Fig. 7 shows a photograph of the experimental setup with the transponder located $4 \mathrm{~m}$ from the radar antennas. The bow-tie antennas are connected to the radar by $3 \mathrm{~m}$ coaxial cables. Figs. 8(a) and 8(b) show the deramped frequency response with the transponder switched off and on, respectively.

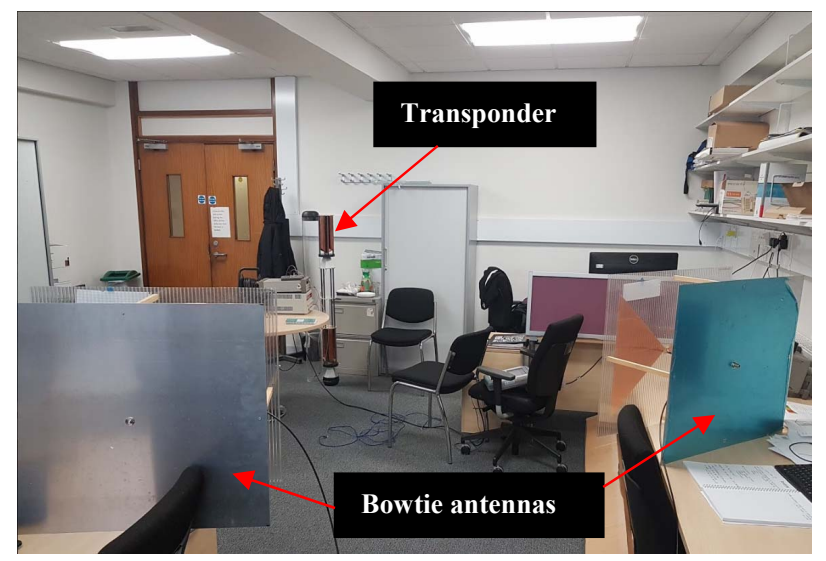

Fig. 7. Indoor measurement setup with transponder and radar.

The $I Q$ data from the FMCW radar was captured using a mixed-signal oscilloscope and processed by a high precision phase-sensitive ranging technique described in [3]. With a frequency-shifted transponder, the desired target signals appear at lower and upper sidebands centred at $f_{\text {mod, }}$, separated by a frequency difference $\Delta_{f}$ that is proportional to twice the distance between the radar and transponder [5]. 


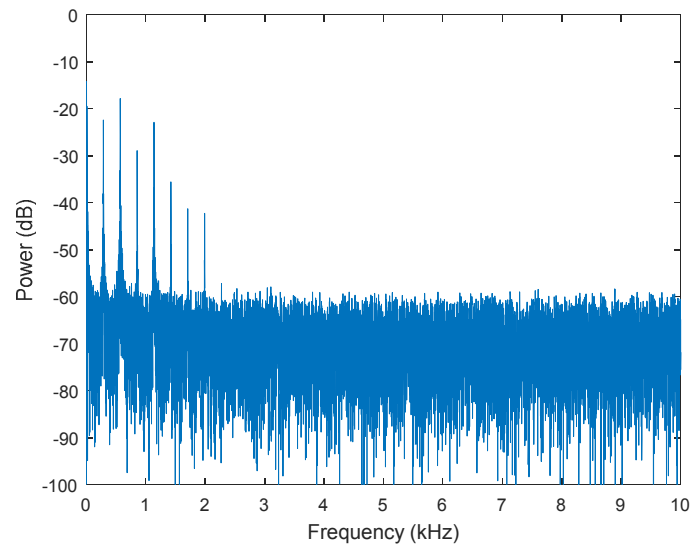

(a)

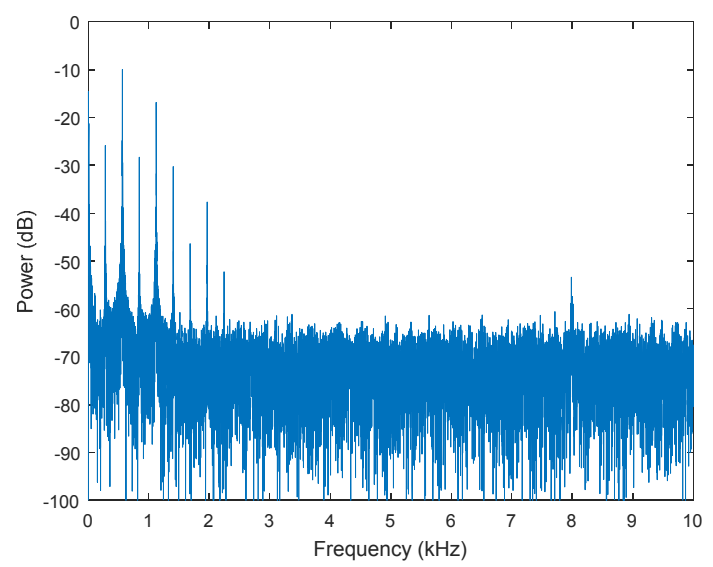

(b)

Fig. 8. Measured radargram with active transponder (a) switched off, and (b) switched on.

The transponder response at $8 \mathrm{kHz}$ is seen clearly and well separated from the stationary targets. Fig. 9(a) shows the upper and lower sidebands centered around $8 \mathrm{kHz}$. Fig. 9(b) shows how $\Delta_{f}$ increases as the distance between the radar and transponder was increased from $1 \mathrm{~m}$ to $4 \mathrm{~m}$.

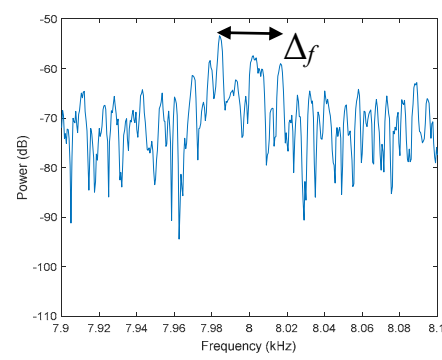

(a)

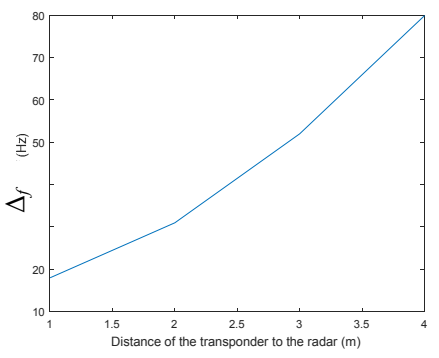

(b)
Fig. 9. (a) Zoomed-in deramped frequency response around $8 \mathrm{kHz}$, and (b) variation of $\Delta \mathrm{f}$ with separation between radar and transponder.

\section{CONCLUSION}

In this paper, a phase-sensitive FMCW radar with active transponder positioning technique is investigated. The basic operation and the proposed technique in locating the transponder in a borehole drilled through ice is explained. An active transponder comprising a frequency-shifted RF electronics unit and two UWB monopole antennas has been developed. The simulated and measured results for transponder antennas are presented. Initial experimental results have shown the position and frequency spectrum of the transponder can be recovered with high accuracy.

\section{ACKNOWLEDGMENT}

This work was funded by the UK Natural Environment Research Council (Grant Number NE/P003281/1). We thank Drs Keith Nicholls and Hugh Corr of the British Antarctic Survey for their technical support of this research.

\section{REFERENCES}

[1] H. Englehardt, N. Humphrey, G. Kamb, M. Fahnstock, "Physical conditions at the base of a fast moving Antarctic ice stream," Science, 248 (4951), pp. 57-59, 1990.

[2] M. J. Siegert et al., "An assessment of deep hot-water drilling as a means to undertake direct measurement and sampling of Antarctic subglacial lakes: experience and lessons from Lake Ellsworth field season 2012/2013," Annals of Glaciology, vol. 55, no. 65, pp. 59-73, 2014.

[3] P. V. Brennan, L. B. Lok, K. W. Nicholls, H. Corr, "Phase-sensitive FMCW radar system for high-precision Antarctic ice shelf profile monitoring", IET Radar, Sonar \& Navigation, 8, pp. 776-786, 2014.

[4] M. Vossiek, R. Roskosch, P. Heide, "Precise 3-D object position tracking using FMCW radar", in $29^{\text {th }}$ European Microwave Conference, Munich, Oct. 1999.

[5] S. Halai, P. V. Brennan, D. Patrick, I. Weller, "Frequency shifted active target for use in FMCW radar systems", in IEEE Radar Conf., Cincinnati, USA, pp. 0819-0823, May 2014. 